\title{
Analysis of Left Ventricular Motion Using a General Robust Point Matching Algorithm
}

\author{
Ning Lin ${ }^{1}$, Xenophon Papademetris ${ }^{2}$, Albert J. Sinusas ${ }^{2,3}$, and James S. Duncan ${ }^{1,2}$ \\ 1 Departments of Electrical Engineering \\ 2 Diagnostic Radiology \\ 3 Medicine, Yale University PO Box 208042, New Haven, CT 06520-8042, USA \\ ning.lin@yale.edu
}

\begin{abstract}
In this paper we present a novel and fully automated approach for the estimation of non-rigid cardiac motion and deformation from sequences of three dimensional medical images. Our approach extends the robust point matching (RPM) algorithm to include shape-based information as inspired by our own previous work in this area. The resulting algorithm labeled as generalized robust point matching (G-RPM) is capable of accurately estimating left ventricular motion without the need of a prior, and often time-consuming, segmentation of the myocardium. We evaluate our approach on both synthetic data as well as using sequences of in-vivo cardiac magnetic resonance images. The approach can easily be adjusted for a number of applications to find the optimal non-rigid transformation.
\end{abstract}

\section{Introduction}

The estimation of regional cardiac motion and deformation has been an active area of research in medical image analysis. In recent years, there have been a variety of efforts aimed at quantitatively characterizing myocardial motion. In general this approaches can be divided into three groups: (i) methods based on magnetic resonance tagging [24 3, 10 [11,8], (ii) methods based on magnetic resonance phase contrast [25 14] and (iii) methods based on computer vision techniques that track tokens in the images using features such as intensity [21], texture [13] or geometric features of extracted surfaces [7,120]. This third group of approaches has the advantage of being applicable to modalities other than magnetic resonance such as echocardiography [17]. For additional information the reader is referred to a review paper by Frangi et al [9] which gives a detailed overview of the current state of the art. A more detailed exposition of individual methods can be also be found in a recently published book [2].

In this work we propose a new method for estimating non-rigid cardiac motion which combines the strengths of our previous work using shape-based tracking [17] and the robust point matching (RPM) framework proposed by Chui and Rangarajan. The shape-based tracking methodology has been successfully used to 2D and 3D nonrigid motion analysis. The main limitation of this methods is that it depends on an accurate segmentation of the myocardium which is still a non-trivial problem. RPM has been shown an effective non-rigid matching approach for several applications [6]. The attractiveness of RPM for the cardiac motion estimation problem is its ability to estimate non-rigid correspondences without the need for a time consuming segmentation step. 
The original RPM framework used a distance metric between corresponding points and is not able to handle outliers in the reference (or template) point set 1

Our approach, described in this paper, extends the RPM framework to (a) use a more general metric form that includes curvature information and (b) handle outliers in both data-sets. This approach can handle more general problems and we name it the Generalized Robust Point Matching (G-RPM) algorithm. In particular the use of shape information is embedded to guide more precise motion recovery and fake feature points or unmatched real feature points are automatically treated as outliers by the G-RPM algorithm during the optimization annealing process. The final outputs are the correspondence and non-rigid transformation between two point-sets at different frames.

\section{Methods}

\subsection{The RPM Algorithm}

The RPM uses fuzzy correspondence with soft-assign and deterministic annealing techniques. Assume we have a data point-set $X=\left\{x_{i}, i=1,2, \cdots, N\right\}$ and a template point-set $Y=\left\{y_{a}, a=1,2, \cdots, K\right\}$. To match set $Y$ onto $X$, it proposed to minimize the following objective energy function:

$$
\begin{aligned}
E(M, \alpha)= & \sum_{i=1}^{N} \sum_{a=1}^{K} m_{a i}\left\|x_{i}-f\left(y_{a}, \alpha\right)\right\|^{2}+\lambda\|\mathcal{L} f\|^{2}-\zeta \sum_{i=1}^{N} \sum_{a=1}^{K} m_{a i} \\
& +T \sum_{i=1}^{N} \sum_{a=1}^{K} m_{a i} \log m_{a i}
\end{aligned}
$$

where $f$ is a non-rigid transformation function with parameters $\alpha . T$ is annealing temperature, gradually decreasing to zero as matching iteration begins. Correspondence matrix $m_{a i} \in[0,1]$ is subject to the constraints: $\sum_{i=1}^{N+1} m_{a i}=1$, for $a \in\{1,2, \cdots, K\}$ and $\sum_{a=1}^{K+1} m_{a i}=1$, for $i \in\{1,2, \cdots, N\}$.

Minimizing eqn. (1) is solved by an alternating update process where two steps are repeated until the algorithm converges. One step is to update the correspondence matrix $m_{a i}$. The closed form solution can be obtained by differentiating the objective function (1) w.r.t. $m_{a i}$ and setting the result to zero:

$$
m_{a i}=e^{-\frac{\left\|x_{i}-f\left(y_{a}, \alpha\right)\right\|^{2}-\zeta}{T}}
$$

A second step to update transformation parameter $\alpha$ involves a least-squared approach given the $m_{a i}$ :

$$
\min _{\alpha} E(\alpha)=\min _{\alpha} \sum_{a=1}^{K}\left\|\hat{x}_{a}-f\left(y_{a}, \alpha\right)\right\|^{2}+\lambda\|\mathcal{L} f\|^{2}
$$

where $\hat{x}_{a}=\sum_{i=1}^{N} m_{a i} x_{i} / \sum_{i=1}^{N} m_{a i}$. This step implies that each point in the template set $Y$ must have the corresponding point in data set $X$.

\footnotetext{
${ }^{1}$ This, strictly speaking, is a limitation of the non-linear implementations of RPM. Earlier implementations which aimed at estimating affine transformations did not suffer from this limitation.
} 


\subsection{The G-RPM Algorithm}

Extensions to RPM can be introduced following the order of RPM's solution procedure. Firstly, consider the case that the optimized result is expected to minimize not only the distance between the corresponding points but also their associated characteristic values, such as intensity, texture and etc. Let us denote these attributes as $\mathcal{A}_{X}\left(x_{i}\right)$ and $\mathcal{A}_{Y}\left(y_{a}\right)$ for the points in set $X$ and $Y$, respectively. The objective function becomes:

$$
\begin{aligned}
E(M, \alpha)= & \sum_{i=1}^{N} \sum_{a=1}^{K} m_{a i}\left[\left\|x_{i}-f\left(y_{a}, \alpha\right)\right\|^{2}+\lambda_{\mathcal{A}} g\left(\left\|\mathcal{A}_{X}\left(x_{i}\right)-\mathcal{A}_{Y}\left(y_{a}\right)\right\|^{2}\right)\right] \\
& +\lambda\|\mathcal{L} f\|^{2}-\zeta \sum_{i=1}^{N} \sum_{a=1}^{K} m_{a i}+T \sum_{i=1}^{N} \sum_{a=1}^{K} m_{a i} \log m_{a i}
\end{aligned}
$$

where $g(\cdot)$ is a strictly increasing function and $\lambda_{\mathcal{A}}$ balances the significance between the distance and new information.

The G-RPM algorithm is designed to solve the general optimization problem of eqn. (4) and at the same time handle the outliers in both sides of point sets. Observing the alternating process style, the generalization takes place in both correspondence matrix $m_{a i}$ update step, and transformation parameters $\alpha$ update step. For $m_{a i}$, it is easy to see the generalized version as:

$$
m_{a i}=e^{-\frac{\left[\left\|x_{i}-f\left(y_{a}, \alpha\right)\right\|^{2}+\lambda_{\mathcal{A}} g\left(\left\|\mathcal{A}_{X}\left(x_{i}\right)-\mathcal{A}_{Y}\left(y_{a}\right)\right\|^{2}\right)\right]-\zeta}{T}}
$$

The double-sided-outlier handler is implemented in the transformation estimation part. Following the scheme proposed by Rohr[18], we weigh each single least-squared term in (3) by a fitting-weight factor, the inverse variance $1 / \sigma_{a}^{2}$. If the variance is high, i.e. the template point $y_{a}$ is uncertain, then less penalty is given to the approximation error at this point. When the variance becomes too high and $1 / \sigma_{a}^{2} \rightarrow 0$, this is equivalent to treat this point as an outlier and take it out of the template set. The new transformation update formula reads

$$
\min _{\alpha} E(\alpha)=\min _{\alpha} \sum_{a=1}^{K} \frac{\left\|\hat{x}_{a}-f\left(y_{a}, \alpha\right)\right\|^{2}}{\sigma_{a}^{2}}+\lambda\|\mathcal{L} f\|^{2}
$$

where $\hat{x}_{a}$ is the same as in (3) and $1 / \sigma_{a}^{2}=\sum_{i=1}^{N} m_{a i}$.

\subsection{System Design for Cardiac Motion Analysis}

Curvature Acquisition and Feature Point Extraction: To date, our shape-based tracking algorithms utilize geometry-based curvature operators: a geometric surface for a region is extracted from the image, and this surface is differentiated with respect to some parameterized space to yield curvatures [5]. However, curvature can directly be expressed in terms of partial derivatives of the gray level images by using area-based operators [19]. For example, the Gaussian curvature $\kappa$ from 3D volume data is given by:

$$
\kappa=\frac{L_{x}^{2} L_{y y} L_{z z}+L_{y}^{2} L_{x x} L_{z z}+L_{z}^{2} L_{x x} L_{y y}}{\left(L_{x}^{2}+L_{y}^{2}+L_{z}^{2}\right)^{2}}
$$




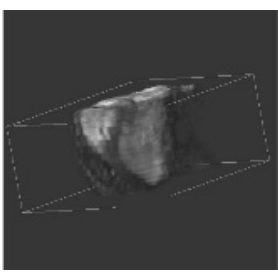

(a)

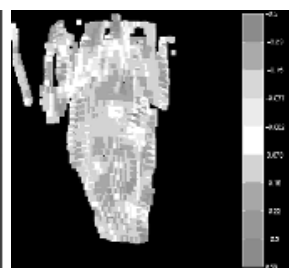

(b)

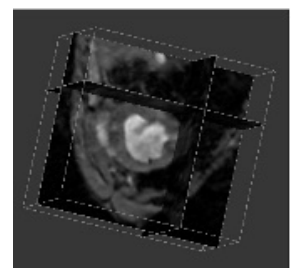

(c)

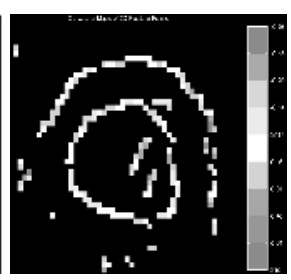

(d)

Fig. 1. (a) Original 3D MRI Data visualized by volume rendering, (c) Orthogonal-slice viewer. Candidate tracking points and their curvature maps: (b) the first principal curvature map for 3D point-set, (d) curvature map for one of its $2 \mathrm{D}$ slices.

where $L(x, y, z)$ denotes image function. $L_{x}, L_{y}$ and $L_{z}$ are the first derivatives of $L$ in $x, y$, and $z$, and $L_{x x}, L_{y y}$ and $L_{z z}$ are the second partials in $x, y$ and $z$.

There are several potential advantages of area-based operators over geometry-based ones. Probably the most important for our application is that an area-based operator is more robust to noise than a geometry-based [12]. Scale space theory has justified the practical use of higher order differential operators, even on noisy images [15]. Analytical study of the behavior of Gaussian derivatives with noise was described in details by [4]. The main result has shown that curvature measurement with certain accuracy can be achieved at an appropriate scale.

Not all the points in images are suitable candidates for tracking. This is because 1) in extrema where the gradient in the denominator of (7) vanishes, the curvature goes to infinity and becomes numerically unstable; 2) shape-based tracking is valid only for points on the boundary. Based on the above considerations, we choose our feature points as follows: 1) to stablize curvature values, discard the points whose edge strength $\|\nabla L\|$ is less than a threshold; 2) thins the wide ridges resulted from the previous step by nonmaximum suppression algorithm [22] and choose the resulted local maxima as the final candidate points to track. An example of the results from our experimental images is shown in figure 1 .

Softassign Design and Non-rigid Mapping Choosing: Adding curvature information into the G-RPM is fairly easy. As in eqn. (5), we replace the general information items $\mathcal{A}_{X}\left(x_{i}\right)$ and $\mathcal{A}_{Y}\left(y_{a}\right)$ with the curvature values $\kappa_{X}\left(x_{i}\right)$ and $\kappa_{Y}\left(y_{a}\right)$. Note that $\kappa$ would be a vector composed of two principal curvatures in 3D case. After matching, we would expect the average curvature difference between corresponding points to be within a small scale. The curvature penalty goes up faster beyond that scale than within it. Thus, the definition of $g(\cdot)$ function in eqn. (5) is employed as $g(x)=x e^{r\left(x-\epsilon_{s}\right)}$, where $\epsilon_{s}>0$ is the pre-defined scale and $r$ is steepness rate. To simulate $g(x)$ decreases with annealing temperature $T$, we could let $r=1 / T$.

Different transformation models can be embedded into the G-RPM algorithm by substituting a specific model for the general function $f$ in eqn. (6). We choose the thinplate spline (TPS) model [23] to parameterize the non-rigid mapping, because it is the only spline that can be clearly decomposed into a global affine and a local non-affine components. To solve the general form of eqn. [6], the solution to the original TPS [23] has been modified [18]. 

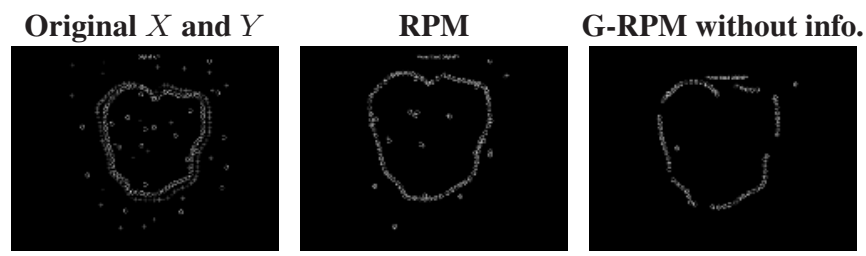

G-RPM with info.
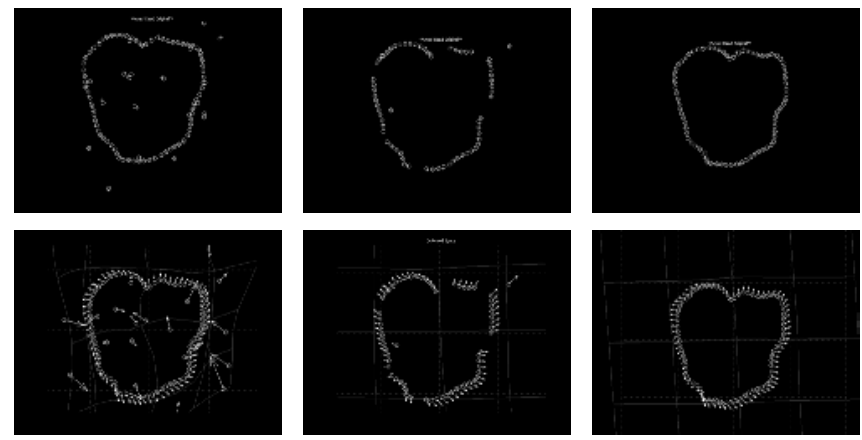

Fig. 2. Top-left: Template (green circle) and target (red cross) point-sets both with noise/outliers. Three methods have been used from left to right: RPM result (first), G-RPM with no other information (second), G-RPM with extra information (third). Top-row: correspondence results. Bottom-row: deformed fields.

\section{Experimental Results}

We test the G-RPM algorithm on 2D synthetic and 3D real point-sets. To solve the problem of different units between intensities and curvatures, we normalize the coordinates of all the point-sets within the same range as the absolute values of curvatures. Since in general, cardiac motion between the adjacent frames is in the range of 0-3 pixels, we set the starting temperature $T_{0}=4$ to be roughly at the same scale (before the normalization). This value is gradually reduced by a factor of 0.90 (annealing rate).

\subsection{Comparison of G-RPM with RPM}

In this experiment, we compare G-RPM with RPM using 2D synthetic data. After two heart-shaped contours are chosen, we add some amount of outliers randomly to each of the point-sets (outlier to original data ratio is about 0.3 in this example). The attribute information $\mathcal{A}$ associated with each point is simulated in this way: original data in both sets have the same values (e.g. 0.1) while outliers are different (e.g. 0.5 and 1.0). Figure 2 shows that although part of outliers can be removed from the target, RPM does not perform well when outliers exist in the template set (each green point in template has to have correspondence in target). G-RPM outperforms RPM and works better because of the additional reliable information source.

\subsection{Application to Cardiac MRI}

We apply the G-RPM to cardiac motion analysis problem with MRI data. Each image data set has 16 frames during one cardiac cycle. The candidate points to track are generated by the technique described in section 2.3 The two principal curvatures are used as the attribute information. The resulted motion fields are illustrated in the top row of figure 3 .

We design two types of methods to validate these results. First, we use expertsegmented contours to check the Euclidean distance error between our warped boundary 

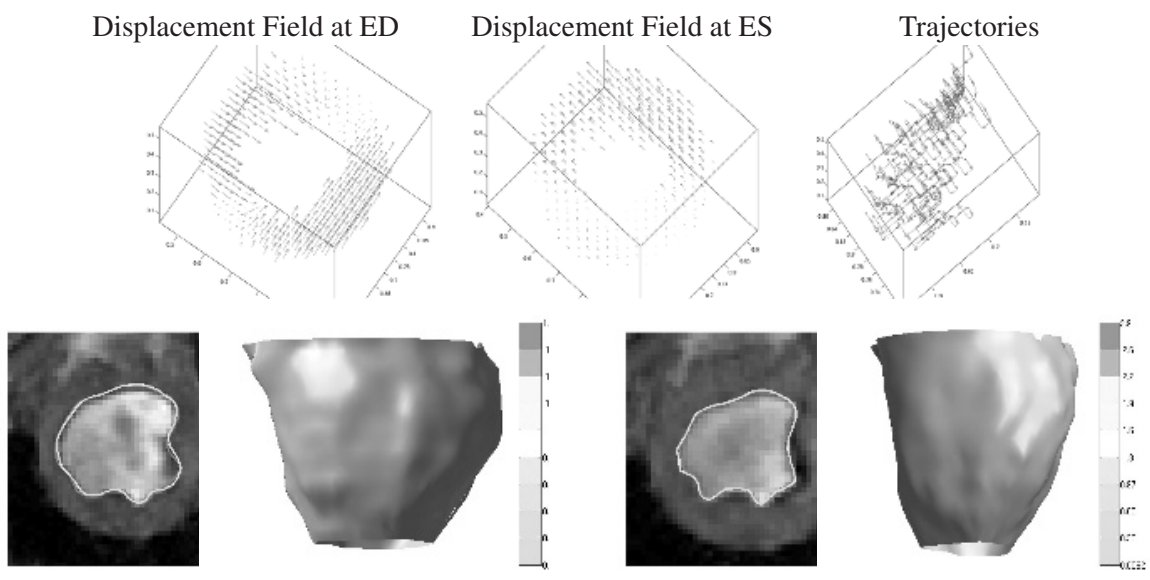

(a) Segmentation ED

(b) Error Map ED

(c) Segmentation ES

(d) Error Map ES

Fig. 3. Results from G-RPM with curvature information. Top: Dense volumetric motion field starting from end-diastole (left) and end-systole (middle). Some of feature points' full-path trajectories over the canine cycle (right). Bottom: (a) and (c) Registration-based segmentations (the red contours are manually pre-defined boundaries and the green are estimated ones). (b) and (d) Pseudo-color mapping of distance error between the warped surface and the true surface .

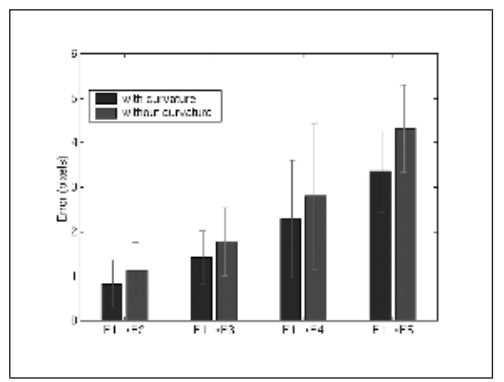

Fig. 4. Absolute displacement error vs implanted markers. The motion is estimated between ED to ES and compared by the GRPM's with and without shape information. The frame-time intervals in $\mathrm{x}$-axis are $1,2,3$ and 4 .

and the true one (figure 3, the bottom row). The experiments show that the estimated boundaries are very close to the expert segmented results (error mean/std: 0.40/0.24 pixel).

To further quantitatively validate resulted motion trajectories, we used 4 canine image sequences with implanted markers for point-by-point displacement comparison (see [16] for more details on the marker implantation and localization.) The marker displacement between ED and ES is estimated in the frame-to-frame manner. The following table summarizes the displacement errors using and not using curvature information.

\begin{tabular}{|l|c|c|c|c|c|c|}
\hline \multirow{2}{*}{} & \multirow{2}{*}{ ED } & \multirow{2}{*}{ ES } & \multicolumn{3}{|c|}{ Error With Curvature (pixel) } & \multicolumn{2}{c|}{ Error Without Curvature (pixel) } \\
\cline { 4 - 7 } & & & mean & std & mean & std \\
\hline subj. 1 & Frame 1 & Frame 6 & 1.4204 & 0.4528 & 1.6326 & 0.6959 \\
\hline subj. 2 & Frame 1 & Frame 5 & 1.2069 & 0.5818 & 1.6258 & 0.4451 \\
\hline subj. 3 & Frame 1 & Frame 8 & 0.9770 & 0.4970 & 0.9854 & 0.5142 \\
\hline subj. 4 & Frame 1 & Frame 8 & 1.9149 & 0.9281 & 2.2096 & 1.0098 \\
\hline average & & & 1.3798 & 0.6149 & 1.6134 & 0.6663 \\
\hline
\end{tabular}


Additionally figure 4 shows displacement errors w.r.t. availability of shape information and time interval between two frames. This experiment illustrates that the errors increase as the frame-to-frame interval becomes larger, while the approach using curvature information always shows the improvement over the other. These results may be explained by the observation that although the larger motion (with a larger time interval) could cause more mismatches, extra useful shape information always benefits the results. It is also interesting to note that relative error reduction by using shape information generally decreases with the time interval (from $26.84 \%, 25.16 \%$ for time interval 1 and 2 to $18.8 \%$ and $22.37 \%$ for 3 and 4 ). This implies that the improvement by using shape information becomes less for larger deformation and thus suggests this technique applies to small deformation scenario. Finally, comparing with the errors from the previous boundarybased tracking technique [20] (where the time interval is 1, average error ( $\mathrm{mean} / \mathrm{std}$ ) 0.99/0.52 pixel for endocardial markers and $0.69 / 0.51$ pixel for epicardial markers), our approach shows a comparable accuracy (average error $0.84 / 0.53$ pixel 2

\section{Conclusion and Future Work}

We presented a new completely automated approach to cardiac motion estimation, which does not require explicit boundary segmentation. The algorithm is robust and can be applied to images contaminated by noise and complicated by missing or diffuse edges. This early experimental results suggest that our new approach has comparable accuracy with traditional shape-based tracking techniques. Further extensions to this work could include the use of different and potentially modality-specific attribute information. Possible examples could include corner-detectors for magnetic resonance tagging and texture information in the case of echocardiography. We are currently investigating the use of alternative transformation models which will allow the incorporation of temporal constraints for multi-frame analysis and explicit biomechanical models (as opposed to thin-plate splines) for the more appropriate regularization of the estimated displacement fields.

\section{References}

1. A. A. Amini and J. S. Duncan. Bending and stretching models for LV wall motion analysis from curves and surfaces. Image and Vision Computing, 10(6): 418-430, 1992.

2. A. A. Amini and J. L. Prince, editors. Measurement of Cardiac Deformations from MRI: Physical and Mathematical Models. Kluwer Academic Publishers, December 2001.

3. L. Axel and L. Dougherty. Heartwall motion: Improved method of spatial modulation of magnetization for mr imaging. Radiology, 172(2): 349-350, 1989.

4. J. Blom, B.M. ter Haar Romeny, A. Bel, and J.J. Koenderink. Spatial derivatives and the propagation of noise in gaussian scale-space. Journal of Visual Communication and Image Representation, 4: 1-13, 1993.

5. M. P. Do Carmo. Differential Geometry of Curves and Surfaces. Prentice Hall Inc., 1976.

6. H. Chui and A. Rangarajan. A new algorithm for non-rigid point matching. In IEEE Conf. Computer Vision and Pattern Recognition, volume 2, pages 44-51. IEEE Press, 2000.

\footnotetext{
${ }^{2}$ Note that the image data used in [20] is different from the data used in this paper.
} 
7. I. Cohen, N. Ayache, and P. Sulger. Tracking points on deformable objects using curvature information. In Lecture Notes in Computer Science-ECCV92, pages 458-466. Springer Verlag, 1992.

8. T. S. Denney Jr and J. L. Prince. Reconstruction of 3-D left ventricular motion from planar tagged cardiac MR images: An estimation theoretic approach. IEEE Transactions on Medical Imaging, 14(4): 625-635, December 1995.

9. A.F. Frangi, W.J. Niessen, and M.A. Viergever. Three-Dimensional modeling for functional analysis of cardiac images: A review. IEEE Trans. Medical Imaging, 20(1): 2-25, 2001.

10. E. Haber, D. N. Metaxas, and L. Axel. Motion analysis of the right ventricle from MRI images. In Medical Image Computing and Computer Aided Intervention (MICCAI), pages 177-188, Cambridge, MA, October 1998.

11. J. Huang, D. Abendschein, V. G. Dávila-Román, and A. A. Amini. Spatio-temporal tracking of myocardial deformations with a 4-D B-spline model from tagged MRI. IEEE Transactions on Medical Imaging, 18(10): 957-972, October 1999.

12. J.J. Koenderink and W. Richards. Two-dimensional curvature operators. Journal of Optical Society of America, 5(7): 1136-1141, 1988.

13. J. Meunier. Tissue motion assessment from 3D echographic speckle tracking. Phys. Med. Biol., 43: 1241-1254, 1998.

14. F. G. Meyer, R. T. Constable, A. G. Sinusas, and J. S. Duncan. Tracking myocardial deformation using spatially constrained velocities. In Information Processing in Medical Imaging. Kluwer, 1995.

15. W. J. Niessen, J. S. Duncan, M. Nielsen, L. M. J. Florack, B. M. ter Haar Romeny, and M. A. Viergever. A multiscale approach to image sequence analysis. Computer Vision and Image Understanding, 65: 259-268, 1997.

16. X. Papademetris, A. J. Sinusas, D. P. Dione, R. T. Constable, and J. S. Duncan. Estimation of 3D left ventricular deformation from medical images using biomechanical models. IEEE Transactions on Medical Imaging, 21(7): 786-800, July 2002.

17. X. Papademetris, A. J. Sinusas, D. P. Dione, and J. S. Duncan. Estimation of 3D left ventricular deformation from echocardiography. Medical Image Analysis, 5(1): 17-29, March 2001.

18. K. Rohr, M. Fornefett, and H. S. Stiehl. Approximating thin-plate splines for elastic registration: Integration of landmark errors and orientation attributes. In Information Processing in Medical Imaging, pages 252-265, Hungary, 1999. Springer.

19. B. M. Ter Haar Romeny, L. M. J. Florack, A. H. Salden, and M. A. Viergever. Higher order differential structure of images. Image and Vision Computing, 12(16): 317-325, 1994.

20. P. Shi, A. J. Sinusas, R. T. Constable, E. Ritman, and J. S. Duncan. Point-tracked quantitative analysis of left ventricular motion from 3D image sequences. IEEE Transactions on Medical Imaging, 19(1): 36-50, January 2000.

21. S. Song and R. Leahy. Computation of $3 \mathrm{D}$ velocity fields from $3 \mathrm{D}$ cine $\mathrm{CT}$ images. IEEE Trans. Medical Imaging, 10: 295-306, 1991.

22. E. Trucco and A. Verri. Introductory Techniques for 3D Computer Vision. Prentice Hall, 1998.

23. G. Wahba. Spline Models for Observational Data. SIAM, Philadelphia, PA, 1990.

24. E.A. Zerhouni, D.M. Parish, W.J. Rogers, A. Yang, and E.P. Shapiro. Human heart: Tagging with MR imaging-a method for noninvasive assessment of myocardial motion. Radiology, 169(1): 59-63, 1988.

25. Y. Zhu, M. Drangova, and N. J. Pelc. Estimation of deformation gradient and strain from cine-PC velocity data. IEEE Transactions on Medical Imaging, 16(6), December 1997. 